\title{
Purification, Expansion and Characterization of Putative Murine Cardiac Progenitor Cells
}

\author{
Kanit Reesukumal ${ }^{1}$, Busadee Pratumvinit ${ }^{1}$, Marianna Rudakova ${ }^{2}$, Kajohnkiart Janebodin ${ }^{3,4}$ and Morayma Reyes ${ }^{2 *}$ \\ ${ }^{1}$ Department of Clinical Pathology, Faculty of Medicine, Siriraj Hospital, Mahidol University, Bangkok, Thailand \\ 2Department of Pathology, University of Washington, Seattle, Washington, United States of America \\ ${ }^{3}$ Department of Oral Health Sciences, University of Washington, Seattle, Washington, United States of America \\ ${ }^{4}$ Department of Anatomy, Faculty of Dentistry, Mahidol University, Bangkok, Thailand
}

\begin{abstract}
Ischemic heart disease kills more people than any other condition. Medical treatment for ischemic heart disease is currently limited by the heart's lack of regeneration after injury. Recent reports have suggested the existence of cardiac progenitor cells in the adult normal and diseased mammalian heart. The origin of these cells is unclear. We implemented novel culturing conditions to isolate putative cardiac progenitor cells ( $\mathrm{pCPCs}$ ) from the adult murine heart atrium with the properties of cardiac regeneration. Putative adult cardiac progenitor cells were purified and expanded from adult murine hearts by expansion medium supplemented with $2 \%$ fetal calf serum, epidermal growth factor, platelet-derived growth factor and leukemia inhibitory factor. Under the culture condition of $5 \% \mathrm{O}_{2}$, these cells can be expanded beyond 42 days, expressed stem cell antigen (Sca-1) and cardiac-specific transcription factors. When treated with oxytocin in vitro, these cells express cardiac contractile proteins and when injected intramuscularly in the tibialis anterior muscle these cells give rise to cardiomyocyte-like cells. In contrast, transplantation of these pCPCs in uninjured heart did not result in cardiomyocyte differentiation suggesting that the heart environment is less permissive than the skeletal muscle of cardiogenesis. These results suggest that cells from the adult murine heart selected with this culture conditions and transplanted in the skeletal muscle have cardiogenic potential. Thus, this approach warrants further investigation for the therapeutic development of strategies for cardiac tissue engineering and myocardial regeneration.
\end{abstract}

Keywords: Cardiac progenitor cells; Sca-1; Oxytocin; Cardiac regeneration

\section{Introduction}

Ischemic heart disease kills more people than any other condition [1]. Current medical treatment can partially improve the outcome of an infarct. Heart transplantation, the only current treatment to replace the lost muscle, is limited by the need for lifelong immunosuppression and limited supply of donor hearts [2,3]. Cell-based therapies are currently being investigated as alternative treatments to achieve cardiac repair [4].

Recent studies have shown that the adult mammalian heart cells can regenerate after injury [5,6]. Several different sources of origin for the cardiac progenitor populations have been examined and characterized. Putative cardiac progenitor populations have been reported including, c-Kit cell surface receptor expressing cells [7], Islet-1 marker expressing cells $[8,9]$, side population cells (SP) $[8,10,11]$, and cells expressing the stem cell antigen receptor $\left(\mathrm{Sca}-1^{+}\right)[12,13]$. It has been suggested that Sca- $1^{+}$cells play a regenerative role following myocardial infarction (MI) based on the observation that the number of endogenous Sca$1^{+}$, CD $31^{-}$cells increased significantly 7 days after MI, as did the expression of Sca-1 protein [9]. Some studies suggest that $\mathrm{c}-\mathrm{Kit}^{+}$and cardiac SP cells in the heart may arise from the bone marrow $[14,15]$.

Although differentiation capacity of the reported cells has been described in terms of protein expression characteristic of cardiogenesis, contractile and energetic compatibility between graft and host is more difficult to determine. A recent study showed poor survival and no long-term functional benefits of cardiac stem cell transplants [16]. We feel that until robust cardiac regeneration can be established with certainty at a functional level, the use of the term "putative" to describe examined cells is prudent. In this report, we therefore confirm the presence of putative cardiac progenitor cells (pCPCs), in the adult murine heart, using a modified culture condition protocol that results in high enrichment of Sca- $1^{+}$cells from the heart atrium [17]. These cells can differentiate in vitro and in vivo into putative cardiomyocytes. Notably, cardiomyocyte differentiation of transplanted cells was only observed during pCPC transplantation into the skeletal muscle, but not the uninjured heart, suggesting that the normal heart environment is less permissive than the skeletal muscle of cardiogenesis.

\section{Materials and methods}

\section{Animals}

Cardiac cell suspensions were prepared from the enhanced green fluorescent protein (GFP) transgenic mice with C57BL/6 background (C57BL/6-Tg (CAG-EGFP) 10sb/J mice, 9-24 weeks of age, $n=3$ ). Animal studies were conducted according to $\mathrm{NIH}$-approved guidelines, as well as approved guidelines of the institutional animal care and use committee of the University of Washington.

*Corresponding author: Morayma Reyes, Department of Pathology, University of Washington, Mercer Street, Room 432, Box 358050, Seattle, WA 98109 United States of America, Tel: +1 206 6165004; Fax: +1 206 8971540; E-mail: morayma@u.washington.edu

Received September 15, 2011; Accepted November 09, 2011; Published November 11, 2011

Citation: Reesukumal K, Pratumvinit B, Rudakova M, Janebodin K, Reyes M (2011) Purification, Expansion and Characterization of Putative Murine Cardiac Progenitor Cells . J Stem Cell Res Ther S1:002. doi:10.4172/2157-7633.S1-002

Copyright: (c) 2011 Reesukumal K, et al. This is an open-access article distributed under the terms of the Creative Commons Attribution License, which permits unrestricted use, distribution, and reproduction in any medium, provided the original author and source are credited. 


\section{Preparation and expansion of pCPCs from the adult murine heart}

Atrium and ventricle heart portions were separated and cardiomyocytes depleted cardiac cell suspensions were prepared as follows. Minced cardiac muscle tissue was digested with $2 \mathrm{mg} / \mathrm{ml}$ collagenase type IV, $1.2 \mathrm{U} / \mathrm{ml}$ dispase II (Worthington, Lakewood, NJ), and $2 \mathrm{mM} \mathrm{CaCl}_{2}$ in $\mathrm{PBS}$ for 45 minutes at $37^{\circ} \mathrm{C}$. Samples were pipetted up and down every 15 minutes to fracture clumps and dissociate mononuclear cells. The digestion was neutralized by adding twice the original volume HAM'S/F10 supplemented with $15 \%$ horse serum (Hyclone, Logan, UT) followed by filtration with a $40 \mu \mathrm{m}$ nylon cell strainers (BD Falcon, Franklin Lakes, NJ) then centrifuged at $300 \mathrm{~g}$ for 5 minutes. To eliminate erythrocytes, the cell pellet was treated with $5 \mathrm{ml}$ hemolytic buffer $\left(155 \mathrm{mM} \mathrm{NH}_{4} \mathrm{Cl}, 10 \mathrm{mM} \mathrm{KHCO}_{2}\right.$, and $0.1 \mathrm{mM}$ EDTA in $\mathrm{H}_{2} \mathrm{O}$ ) for 4 minutes at room temperature. Cell suspensions were counted and centrifuged again at $300 \mathrm{~g}$ for 5 minutes. $2 \times 10^{5}$ cells were plated in $5 \mathrm{ml}$ expansion medium on standard polystyrene tissue culture dishes (BD Falcon, Franklin Lakes, NJ). Expansion medium consisted of $60 \%$ low glucose DMEM (Hyclone, Logan, UT), 40\% MCDB 201, 2\% fetal calf serum (FCS), 1X insulin transferrin selenium (ITS), $1 \mathrm{X}$ linoleic bovine serum albumin (LA-BSA), $10^{-9} \mathrm{M}$ dexamethasone, and $10^{-4} \mathrm{M}$ ascorbic acid 2-phosphate (all from Sigma, St. Louis, MO), $100 \mathrm{U}$ penicillin, $1000 \mathrm{U}$ streptomycin (Hyclone, Logan, UT), supplemented with $1 \times 10^{3}$ units/ml leukemia-inhibitory factor (LIF-ESGRO, Millipore), $10 \mathrm{ng} / \mathrm{mL}$ EGF (Sigma) and $10 \mathrm{ng} / \mathrm{mL}$ PDGF-BB (R\&D). Cultures were incubated at $37^{\circ} \mathrm{C}, 5 \% \mathrm{CO}_{2}$ and $5 \% \mathrm{O}_{2}$. Media was initially changed at day three and subsequently replaced every three to four days or with every passage to a new dish. Putative cardiac progenitor cell (pCPC) monolayers were passaged when 60 $80 \%$ confluency was reached at cell density of 5000 cells $/ \mathrm{cm}^{2}$. Cell transplantations and differentiations were set up at passage 10 .

\section{Reverse transcription- polymerase chain reaction for} amplification of cardiomyocyte genes

Total RNA was isolated using RNeasy column (Qiagen, Valencia, CA) with RNase-free DNase treatment. First strand cDNA was synthesized using the High Capacity cDNA synthesis kit from Applied Biosystems (Foster City, CA) per manufacturer's instructions. Primers for cardiomyocyte gene amplification were as follows. Glyceraldehyde3-phosphate dehydrogenase (GAPDH): sense, 5'-CTC GTC CCG TAG ACA AAA TGG-3' , antisense, 5'-CGC TCC TGG AAG ATG GTG-3'; GATA-4: sense, $5^{\prime}$-CCC GGG CTG TCA TCT CAC TA-3', antisense, 5'-GTG CCC CAG CCT TTT ACT TTG-3'; GATA-6: sense, 5'-AGC AGG ACC CTT CGA AAC G-3', antisense, 5'-GCG CTT CTG TGG CTT GAT G-3'; Nkx2.5: sense, 5'-CCA AGT GCT CTC CTG CTT TCC-3', antisense, 5'-AGG TAC CGC TGT TGC TTG AAG-3'; C-kit: sense, 5'-AGC CTG GCG TTT CCT ACG T-3', antisense, 5'GCC CGA AAT CGC AAA TCT TT-3'; Oct4: sense, 5'-CTG GGC GTT CTC TTT GGA AA-3', antisense, 5'-TCG GGC ACT TCA GAA ACA TG-3'; Nanog: sense, 5'-TCT CAA GTC CTG AGG CTG ACA AG-3', antisense, 5'-GTG CTG AGC CCT TCT GAA TCA-3'; Klf4: sense, 5'-GGT TTT GGT TTG AGG TTT TGT TTC T-3', antisense, 5'-CCT CAC GCC AAC GGT TAG TC-3'; Myh6: sense, 5' - AGC CCA GTA CCT CCG AAA GTC-3', antisense, 5' - CGT GCA GGA AGG TCA GCA T-3'; cTnI: 5'- ATT GCG AAG CAG GAG ATG GA-3', antisense, 5' - ACT TGC CAC GGA GGT CAT AGA-3'; vWF: 5'-GAT GTC CAG CTC CCC TTC CT-3', antisense, 5'-AGG CGT TTC CGA AGT CTA CCA-3'; albumin: 5'-TCA ACT GTC AGA GCA GAG AAG C-3', antisense, 5'-AGA CTG CCT TGT GTG GAA GAC T-3'. The
PCR cycling conditions were as follows; $95^{\circ} \mathrm{C}$ for 7 minutes: 35 cycles at $95^{\circ} \mathrm{C}$ for $30 \mathrm{sec}, 55^{\circ} \mathrm{C}$ for $30 \mathrm{sec}$ and $72^{\circ} \mathrm{C}$ for $45 \mathrm{sec}$. PCR products were ran on $2 \%$ agarose gels.

\section{Characterization of cultured pCPCs by flow cytometry analysis}

The pCPCs grown in culture were trypsinized with $0.25 \%$ trypsin-EDTA (HyClone, Logan, UT) at $37^{\circ} \mathrm{C}$ for 5 minutes then the enzymatic reaction was inhibited with FCS $10 \%$ volume of the trypsin used. Cells were centrifuged and resuspended in $1 \mathrm{ml}$ PBS (HyClone, Logan, UT) then counted and transferred to $1.7 \mathrm{ml}$ Eppendorf tubes for staining with eFluor ${ }^{\mathrm{TM}} 605^{\mathrm{NC}}$ (Q.605) anti-mouse Ly-6A/E (Sca1), Allophycocyanin-eFluor ${ }^{\mathrm{TM}} 780$ (APC Cy7) anti-mouse CD117 (c-Kit) (all from eBioscience, San Diego, CA) and permeabilized with $2 \%$ PFA then $0.3 \%$ Saponin before staining with Alexa Fluor ${ }^{\infty} 647$ anti-mouse Nanog (eBioscience, San Diego, CA) or polyclonal goat anti- Nkx2.5 (Santa Cruz Biotechnology, Santa Cruz, CA) followed by anti-goat Alexa 647 conjugated antibody (Invitrogen, Carlsbad, CA). Antibodies were diluted and combined in PBS $+0.3 \%$ bovine serum albumin (BSA) at a ratio of $600 \mathrm{ng}$ of each antibody per $10^{6}$ cells, except for Sca-1 antibody, which was added at $300 \mathrm{ng}$ per $10^{6}$ cells due to its strong avidity. $10^{5}$ cells per single color controls were stained with 200 ng of each antibody/fluorophore along with one containing only cells for unstained control. Samples and controls with antibodies in PBS + $0.3 \%$ BSA were incubated on ice in the dark for 45 minutes. Cells were washed and centrifuged for 5 minutes at $300 \mathrm{~g}$ for unpermeabilized cells or $800 \mathrm{~g}$ for permeabilized cells then resuspended in PBS+0.3\% BSA.

FACS- analysis was performed using a BD FACSAria II (BD Biosciences, San Jose, CA). Software compensation of single color controls were used to correct the potential spectral overlap between color filters and fluorophores. The cells positive for Sca-1, c-kit, Nanog, and Nkx2.5 were analyzed by the FlowJo v7.54 (TreeStar, Ashland, OR).

\section{Immunostaining of cultured pCPCs}

Immunofluoroscence staining was done using a monoclonal $\mathrm{Cy} 3$ conjugated anti-

SMA Ab (Sigma, St. Louis, MO) at 1:500 dilution, monoclonal rat anti-Sca-1 antibody (BD Pharmingen, San Jose, CA), at a 1:400 dilution, followed by anti-rat Alexa 594 conjugated antibody (Invitrogen, Carlsbad, CA) at 1:1000 dilution.

\section{Immunohistochemistry staining for terminally differentiated cardiomyocytes in cultured pCPCs}

Anti-MF-20 (cardiac sarcomeric myosin) Ab (Developmental Studies Hybridoma Bank, Iowa), incubated overnight at $4^{\circ} \mathrm{C}$, and polyclonal goat anti-Nkx2.5(Santa Cruz Biotechnology, Santa Cruz, $\mathrm{CA}$ ), at 1: 50 dilution, were used as primary antibody followed by biotinylated anti-mouse or goat antibody (Sigma, St. Louis, MO) at 1:200 dilution, and treatment with $\mathrm{ABC}$ kit Vectastain PK-6100 and 3,39-diaminobenzidine (DAB) substrate kit, according to manufacturers protocol (Vector Laboratories, Burlingame, CA). Avidin/biotin block was administered prior to staining with the biotinylated anti-mouse and goat antibody.

\section{Animal surgery and cell transplantation}

Two types of transplant studies were conducted; cultured cells directly injected intramuscularly in the tibialis anterior (TA) muscle 
and the heart. Mice were anesthesized with isofluorane. $10^{6}$ cultured pCPCs resuspended in $30 \mu \mathrm{l}$ PBS were directly injected into the TA muscle of 2-month-old C57BL6 mice $(n=8)$ and $2 \times 10^{6}$ cultured cells in $80 \mu \mathrm{l}$ PBS were directly injected into the heart of 12-monthold C57BL6 mice $(n=3)$. All mice were sacrificed 2 weeks after the injection. Euthanasia and animal injections were in compliance with local guidelines and protocols approved by the Institutional Animal Care and Use Committee.

\section{Immunofluorescent staining of transplanted tissues}

$10-\mu \mathrm{m}$ cryosections of transplanted tissue embedded in optimal cutting temperature compound (OCT) on glass slides were stained sequentially for GFP and troponin-T and were also stained with 49,6-Diamidino-2-phenylindole dihydrochloride (DAPI), purchased from Vector Labs (Burlingame, CA). Polyclonal goat anti-GFP Ab (ABCAM, Cambridge, MA) was used at 1: 100 dilution followed with anti-goat Alexa 488 antibody (Invitrogen, Carlsbad, CA) diluted at 1:1000. Polyclonal rabbit anti-troponin-T Ab (ABCAM, Cambridge, MA) was used at 1: 1000 dilution, followed with anti-rabbit Alexa 594 antibody (Invitrogen, Carlsbad, CA) diluted at 1:1000. Polyclonal rabbit anti-connexin $43 \mathrm{Ab}$ (ABCAM, Cambridge, MA) was used at 1: 500 followed with anti-rabbit Alexa 594 antibody (Invitrogen, Carlsbad, CA) diluted at 1:1000. All immunofluorescence described in this manuscript was detected using a Zeiss Axiovert 200 fluorescent microscope (Thornwood, NY). Photographs were taken with an onboard monochrome AxioCam MRm camera and colored using Adobe Photoshop (San Jose, CA).

\section{Masson's trichrome staining of transplanted tissues}

$10-\mu \mathrm{m}$ cryosections of transplanted tissue embedded in optimal cutting temperature compound (OCT) on glass slides were stained for $3 \mathrm{~min}$ in $50 \%$ hematoxylin solution (Protocol $^{\circledR}$ Fisher Scientific, Kalamazoo, MI), rinsed in tap water, stained for $4 \mathrm{~min}$ in Biebrich scarlet-acid fuchsine (Sigma-Aldrich, St. Luis, MO), rinsed in $0.5 \%$ acidified water ( $5 \mathrm{ml}$ glacial acidic acid per $1 \mathrm{~L} \mathrm{H}_{2} \mathrm{O}$ ), immersed for 10 min in PMA-PTA solution (5ml Phosphomolybdic acid $10 \% \mathrm{w} / \mathrm{v}, 5 \mathrm{ml}$ Phosphotungstic acid per $10 \mathrm{ml} \mathrm{H}_{2} \mathrm{O}$ - reagents from Sigma-Aldrich, St. Luis, $\mathrm{MO}$ ), rinsed in acidified water, stained for $4 \mathrm{~min}$ in $2 \%$ fast green solution (in $195 \mathrm{ml} \mathrm{H}_{2} \mathrm{O}$ and $2 \mathrm{ml}$ glacial acetic acid, $4 \mathrm{~g}$ fast green FCF from Sigma-Aldrich, St. Luis, MO), rinsed in 2 changes of acidified water, dehydrated in 3 changes of $100 \%$ ethanol and cleared in xylene. Prior to light-microscopy analysis, stained slides were covered with cover slips using a resinous mounting medium.

\section{In vitro differentiation of $\mathrm{pCPCs}$ with oxytocin}

To induce cardiomyocyte differentiation, we used oxytocin treatment as previously described by Matsuura et al. [18]. In brief, pCPCs in the density of $2 \times 10^{4}$ cells per $\mathrm{cm}^{2}$ were cultured on $1 \%$ gelatin-coated dishes with Dulbecco's Modified Eagle Medium (DMEM) supplemented with 10\% FBS, $100 \mathrm{U}$ penicillin, and $1000 \mathrm{U}$ streptomycin at $37^{\circ} \mathrm{C}$ in humidified air containing $5 \% \mathrm{CO}_{2}$. Twentyfour hours after seeding, the cells were treated with $100 \mathrm{nM}$ oxytocin (RayBiotech, Norcross, GA). After treatment, the medium was changed every three to four days. Total time in culture was 21 days. RT-PCR from RNA extractions and immunohistochemistry staining were used to determine the extent of cardiomyocytes differentiation. pCPCs cultured in the expansion medium without oxytocin served as control.
In vitro differentiation of $\mathrm{pCPCs}$ with vascular endothelial growth factor (VEGF) and fibroblast growth factor 4 / hepatocyte growth factor (HGF)

Three pCPCs lines were exposed to VEGF or FGF4 and HGF to determine their capacity to differentiate into endothelial and hepatocyte-like cells respectively as previously described $[19,20]$. pCPCs were seeded at a density of $2 \times 10^{4}$ cells per $\mathrm{cm}^{2}$ in 24 -well plates in expansion medium overnight. After 12 hours, pCPCs formed an adherent monolayer, expansion medium was removed, cell monolayers were washed with PBS and medium was replaced by serum free medium containing 60\% low glucose DMEM (Hyclone, Logan, UT), 40\% MCDB 201, 1X insulin transferrin selenium (ITS), 1X linoleic bovine serum albumin (LA-BSA), $10^{-9} \mathrm{M}$ dexamethasone, and $10^{-4}$ $\mathrm{M}$ ascorbic acid 2-phosphate (all from Sigma, St. Louis, MO), $100 \mathrm{U}$ penicillin, $1000 \mathrm{U}$ streptomycin (Hyclone, Logan, UT)) supplemented with $10 \mathrm{ng} / \mathrm{mL}$ VEGF (R\&D) for endothelial differentiation or 10ng/ mL FGF4 and 20ng/mL HGF (R\&D) for hepatocyte differentiation. Cultures were incubated at $37^{\circ} \mathrm{C}, 5 \% \mathrm{CO}_{2}$ and $5 \% \mathrm{O}_{2}$ and respective media were changed every 4 days for 14 days.

\section{Results}

\section{pCPCs proliferated and expanded beyond 45 days in culture}

Cardiomyocytes depleted cardiac cell suspensions in the expansion medium did not survive after 2 weeks in atmospheric oxygen conditions $\left(20 \% \mathrm{O}_{2}\right)$. Under physiological hypoxia $\left(5 \% \mathrm{O}_{2}\right)$, many cells died early in culture, however, after the first week of culture, spindle like cells that form colonies were observed. These cells were later termed putative cardiac progenitor cells (pCPCs). The colonies from the atrium proliferated at a rate of 13-15 cell divisions within 40 days and were viable beyond 42 days in culture. However, the cells from the ventricle proliferated slower and reached senescence early in culture (Figure 1). Therefore all subsequent studies described herein were done with pCPCs derived from atrium.

\section{Cultured cells express Sca-1, smooth muscle actin and cardiac transcription factors}

pCPCs grown in expansion medium stained positive for Sca-1

Cardiac Progenitor Cell Doubling vs Time

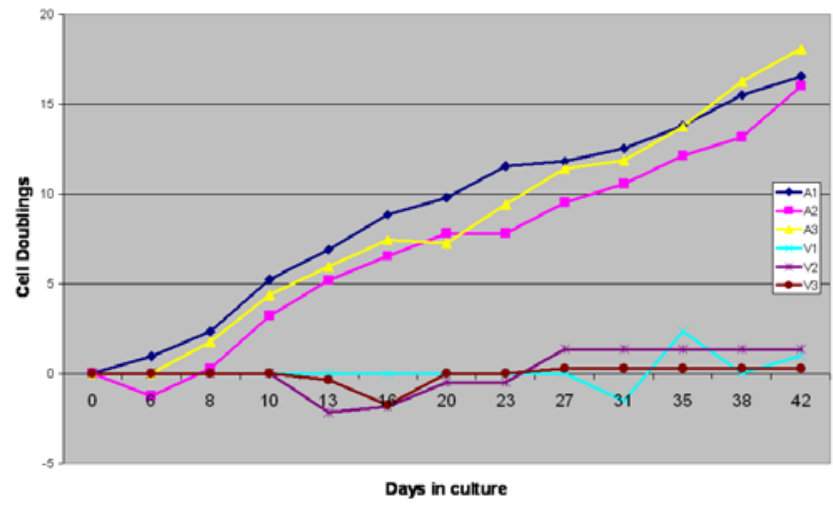

Figure 1: pCPCs can be cultured and expanded. Growth rate comparison between 6 different cell lines derived from atrium and ventricle of 3 individual mice age 3, 4 and 6 months. pCPCs from the atrium (A1, A2, and A3) cultured in expansion medium began to proliferate after day $7^{\text {th }}$ and expanded beyond 40 days but not the cells from ventricle (V1, V2, and V3), which did not grow well. 
and smooth muscle actin (SMA) at day 40 (supplementary figure 1). RT-PCR of cultured cell at the beginning (passage 0 ) slightly express Klf4, GATA- 4 and GATA- 6 while the cultured cells (after 42 days) had increased Klf4, GATA-4 and GATA- 6 expression and also expressed Nanog, c-kit and Nkx2.5. Early and late cultures were always negative for the pluripotent transcription factor Octamer-4 (Oct4) and cardiac myosin heavy chain, Myh6 (Figure 2).

\section{pCPCs are Sca- $1^{+}$cells by flow cytometric analysis}

Flow cytometric analysis of pCPCs after 6 weeks in expansion medium culture demonstrated majority of the cells with Sca-1, Nanog and Nkx2.5 expression, while c-Kit was only slightly expressed (supplementary figure 2). pCPCs were also positive for $\mathrm{Nkx} 2.5$ by staining (Figure 3A).

\section{Oxytocin-treated pCPCs expressed cardiomyocyte properties in vitro}

pCPCs supplemented with oxytocin in the expansion media showed induction of morphological changes including cellular arrangement and elongation. The control cells, pCPCs without oxytocin in the expansion medium, did not show these morphological changes (supplementary figure 3 ). Immunostaining confirmed the induction of cardiac sacromeric myosin (MF-20) in oxytocin-treated cells but not in the control cells (Figure 3B), although the staining pattern was perinuclear which suggest an immature phenotype [21]. We examined the gene expression of cardiac transcription factors and cardiac structural proteins by RT-PCR. Before treatment with oxytocin, only Nkx2.5 and GATA4 cardiac transcription factors were slightly expressed. Cardiac structural protein, troponin-I, was expressed after three weeks of oxytocin treatment (Figure 4). However, cardiac myosin heavy chains were not expressed (not shown) also consistent with an immature phenotype.

\section{Failure of pCPCs to differentiate into non-cardiomyocyte lineages}

pCPCs after 6 weeks in expansion medium were induced to

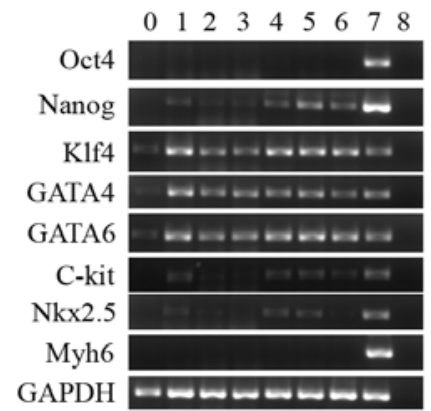

Figure 2: RT-PCR analysis of pCPCs in culture. Cells expressed Klf4, GATA4 and GATA6 cardiac transcription factors slightly at the beginning with increased expression after prolonged time in expansion culture. Nanog and c-kit were expressed slightly at 2 weeks and significantly after 6 weeks expansion culture. Nkx2.5 expressed in 2 of the 3 cell lines at 6 weeks expansion culture. None of the cells expressed Oct4 or Myh6.

Lane $0=$ cells passage 0 at the beginning of the culture. Lanes $1-3=$ three different cell lines at 2 weeks under $5 \%$ oxygen culture in the expansion medium. Lanes $4-6=$ three different cell lines at 6 weeks after culture. Lane 7 = positive control; embryonic stem cells for pluripotent genes (Oct4, Nanog and Klf4) and neonatal heart for the other genes. Lane $8=$ negative control; no template.

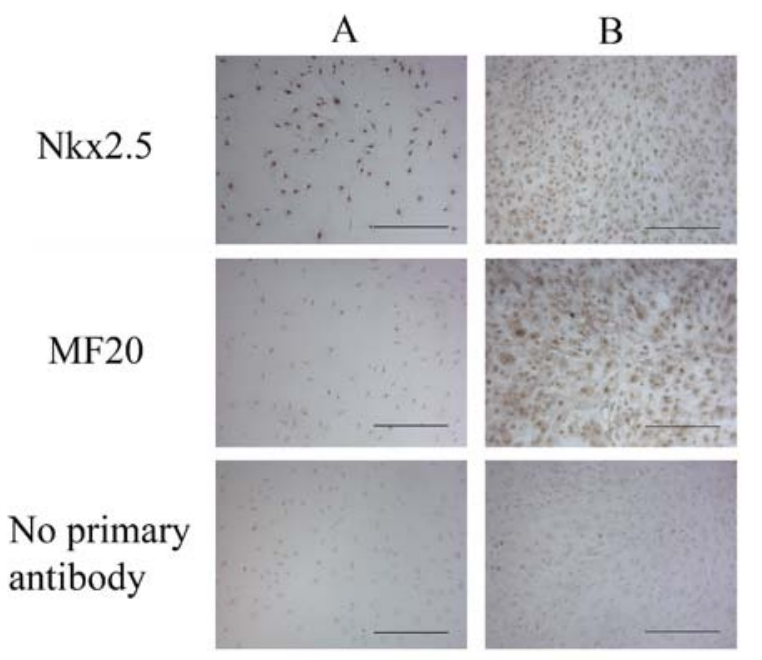

Figure 3: Immunohistochemistry of cells supplemented with oxytocin suggested pCPC differentiation into immature cardiomyocytes (A) Immunostaining showed $\mathrm{Nkx} 2.5^{+}$cells but no cardiomyocyte protein (MF-20 $\left.0^{+}\right)$ in pCPCs cultured with expansion medium. (B) pCPCs express sarcomeric myosin (MF-20) after 21 days of oxytocin treatment. Scale bars $=500 \mu \mathrm{m}$

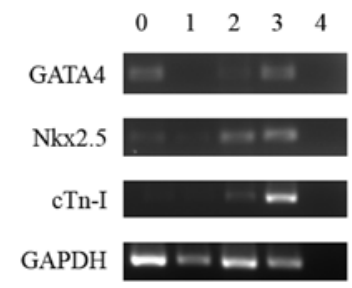

Figure 4: RT-PCR analysis of cardiac genes. pCPCs before treatment with oxytocin (lane 0) expressed GATA4 and Nkx2.5 slightly. None of the cells in expansion medium at day 21 (lane 1) expressed any cardiac transcription factors. After 21 days treatment with oxytocin (lane 2), pCPCs down-regulated GATA4, up-regulated Nkx2.5 and expressed troponin-I. Neonatal heart (lane 3) and no template control (lane 4) were used as positive and negative controls, respectively.

differentiate into endothelial-like and hepatocyte-like cells. 14 days after exposure to differentiation media cells were analyzed by RTPCR for the endothelial marker, von Willebrand Factor (vWF); and the hepatocyte marker, albumin, respectively. All three induced pCPC lines were negative for vWF and albumin (supplementary figure 4).

\section{pCPCs differentiated into cardiomyocyte-like cells when transplanted in skeletal muscle}

The atrium-derived pCPCs from GFP transgenic mice, which were transplanted into the skeletal muscle of wild type mice, formed new muscular bundles 14 days after transplant. These GFP positive muscular bundles were not positive for skeletal specific myosin (data not shown), however, they stained positive for cardiac Troponin-T and their sizes were smaller than the endogenous mature muscular bundles from the skeletal muscle (Figures 5A-B). Untransplanted skeletal muscles were negative for cardiac Troponin- $\mathrm{T}$ staining, indicating the cardiac specificity of this antibody. In contrast to the cytoplasmic GFP pattern in transplanted cells at injection tract (Figures 5A-B, arrow) which were not Troponin- $T$ positive, the GFP pattern in Troponin- $T$ positive bundles was cross-striated, which has been previously described in transgenic cardiomyocytes expressing GFP driven by cardiac-actin 

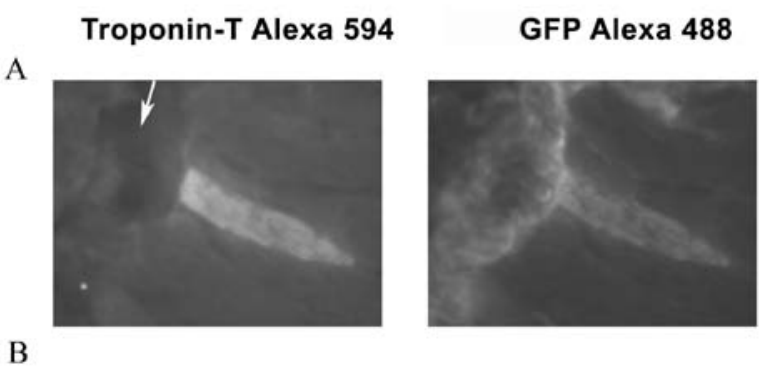

\section{Merged with DAPI}
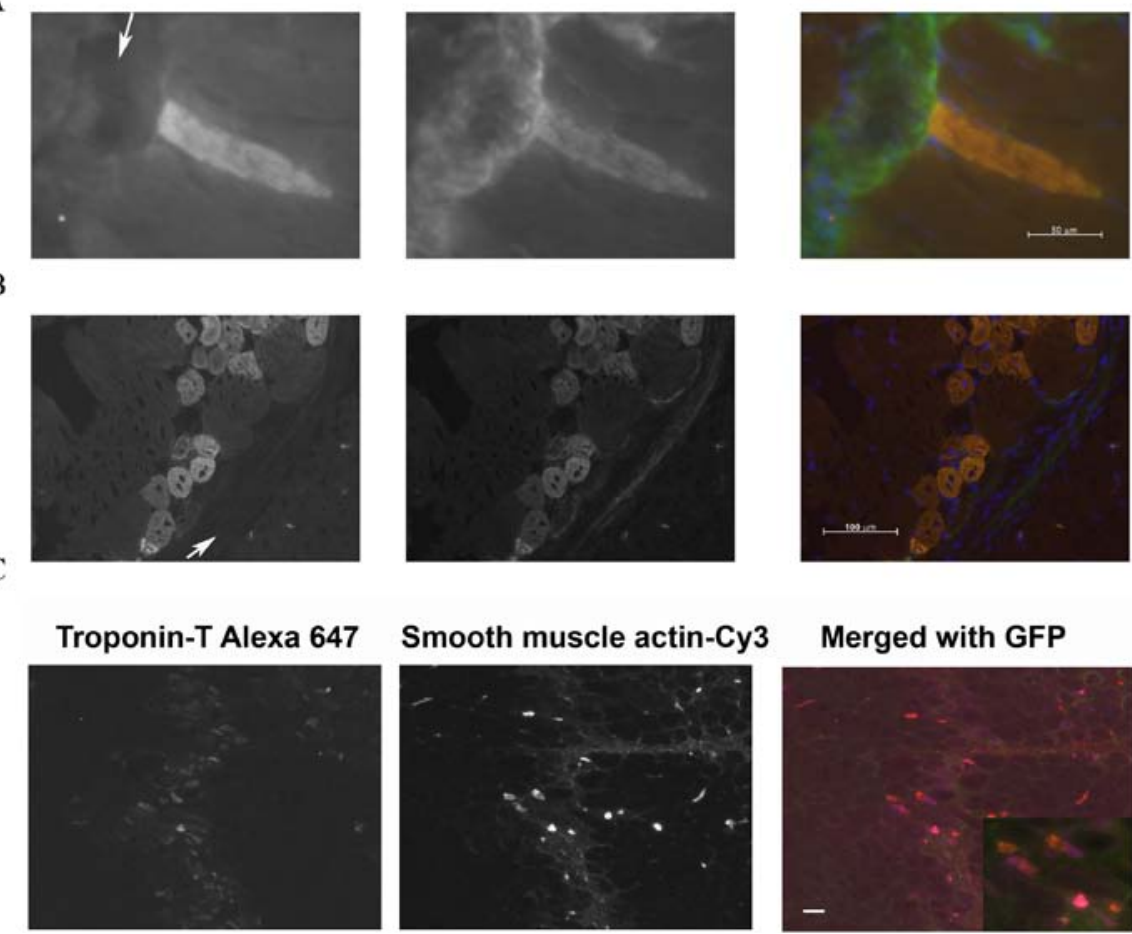

\section{Merged with GFP}

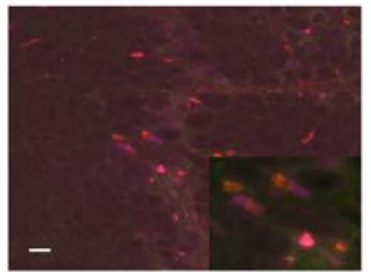

Figure 5: pCPCs gave rise to cardiomyocyte-like cells in skeletal muscle 14 days after transplantation. (A) High magnification of skeletal muscle transverse sections showed small muscular bundles with cross striation positive for cardiac Troponin-T in red (Alexa 594) co-localizing with anti-GFP in green (Alexa 488). (B) Crosssectional sections showed GFP positive small muscular bundles with co-expression of cardiac Troponin-T. The arrow indicated an injection tract. (C) Co-expression of cardiac Troponin-T in magenta (Alexa 647) and smooth muscle actin in red (Cy3) was observed in some of the transplanted GFP+ muscular bundles. The inset showed a higher magnification of the center field where co-localization of cardiac Troponin-T and smooth-muscle actin was observed in some muscular bundles whereas in others transition from adjacent smooth-muscle actin positive into cardiac Troponin-T positive bundles was also observed. Scale bars $=50 \mu \mathrm{m}(\mathrm{A}$ and $\mathrm{C}), 100 \mu \mathrm{m}(\mathrm{B})$

promoter [22]. Some Troponin+/GFP+ muscular bundles were binucleated which is commonly seen in cardiomyocytes (Figure 5B). In addition, these new muscular bundles were positive for smooth muscle actin, which was expressed by pCPCs in culture. Furthermore, transition of smooth muscle actin positive muscular bundles that coexpressed cardiac Troponin-T is seen in the transplanted cells (Figure 5C), indicating progression from early cardiogenic markers to more mature markers $[23,24]$. The cross-striation of the Troponin+/GFP+ muscular bundles differ from skeletal muscle myofibers and unlike the skeletal muscle fibers which show a continuum syncytium of myonuclei along the muscle fibers, the Troponin+/GFP+ muscular bundles seem to have gap junctions connecting them (Figure 5A). Thus we stained for connexin 43 , a gap junction protein expressed in cardiac intercalated disks, which was positive in the cell-cell membrane boundaries exclusively in the transplanted tissue that co-stained with GFP and Troponin-T (Figure 6).

\section{Transplantation of pCPCs in heart resulted in fibrotic tissue} at the injection site

Due to autofluorescence of the heart tissue specially in necrotic and infarcted areas [25], prior to transplantation we labeled the GFP+ pCPCs with PKH26, a red fluorescent dye that irreversibly binds the cell membrane and has successfully been used for in vivo transplantation of the cells [26]. Using this double tracking system, we found GFP+/ $\mathrm{PKH} 26^{+} \mathrm{pCPCs}$ in the needle track surrounded by fibrotic tissue 14 days after transplantation into the heart (Figure 7). These GFP+PKH-26+ cells did not acquire the morphology of mature cardiomyocytes and did not stain positive for cardiac troponin (Figures 7 G-N, inset in $\mathrm{M}$ and $\mathrm{N}$ ), indicating that in contrast to the skeletal muscle transplant, pCPCs transplanted in the heart did not differentiate into cardiomyocytes but the injection elicited a fibrotic response which was also observed in the mock injected hearts. It is likely this fibrotic response impaired the capacity of pCPCs to undergo cardiogenesis in the heart.

\section{Discussion}

We describe a new culture method for selection of the putative cardiac progenitor cells from the adult murine heart. This method is a modification of previous methods used to isolate and cultureexpand stem cells from the bone marrow, including multipotent adult progenitor cells (MAPC), multipotent adult stem cells (MASC) and marrow-isolated adult multilineage inducible (MIAMI) cells [17,2729]. Using the expansion medium containing $2 \%$ FCS, EGF, PDGFBB, Beltrami et al. [29] successfully isolated human multipotent cells from the heart, which expressed undifferentiated cell marker Octamer-4 (Oct4) with the capacity to differentiate into 3 germ layers. Unlike Beltrami et al, we isolated cells which did not express Oct4 but were positive for other pluripotent stem cell genes such as Nanog and KLF4. Mononuclear cardiac cells did not express Nkx2.5 and c-kit immediately after isolation but rather up-regulated the expression of Nkx2.5 and c-kit over time in culture. This indicates first that we did not have contamination of cardiomyocytes or committed cardiac progenitor cells early in culture, and second, that up-regulation of 
Citation: Reesukumal K, Pratumvinit B, Rudakova M, Janebodin K, Reyes M (2011) Purification, Expansion and Characterization of Putative Murine Cardiac Progenitor Cells . J Stem Cell Res Ther S1:002. doi:10.4172/2157-7633.S1-002

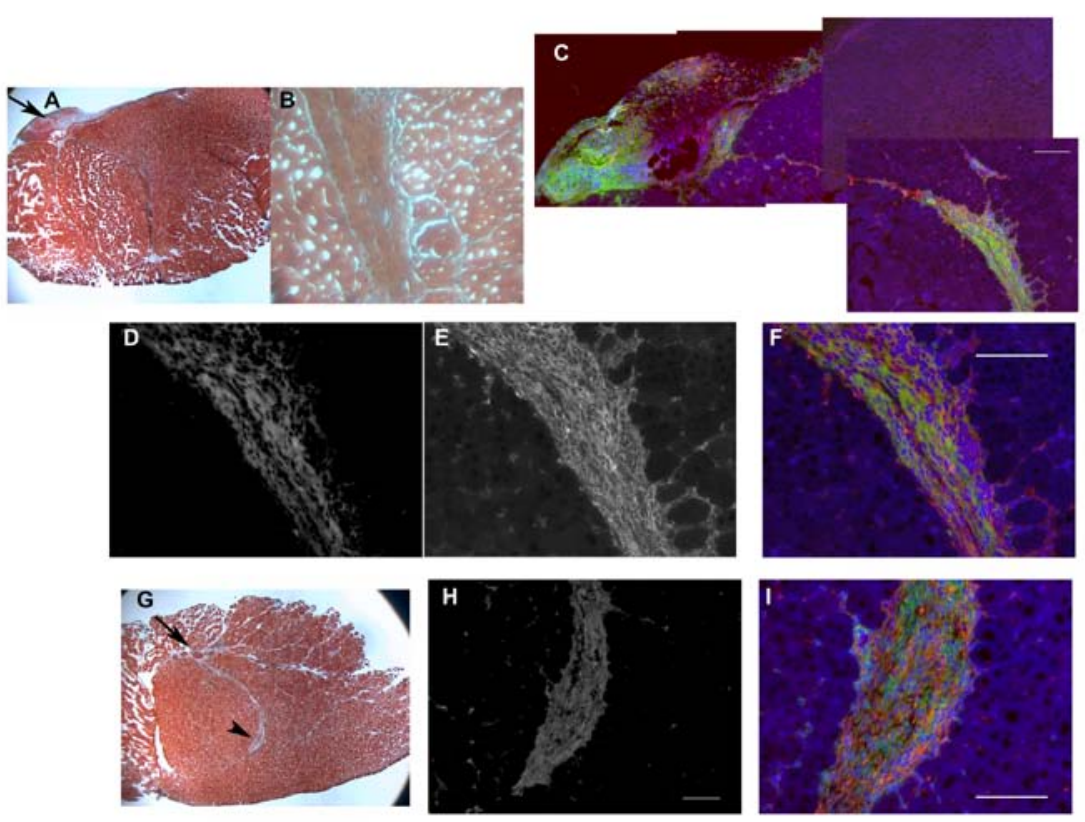

Figure 6: $\mathrm{pCPCs}$ transplanted in skeletal muscle formed muscle bundles that expressed connexin 43 , the gap junction protein expressed in cardiac intercalated disks. (A and B) Masson's trichrome staining of the transplanted TA muscle showed pCPC transplanted cells in the injection track (pointed by arrow) forming muscle like tissues (B). Notice that pCPCs did not form fibrotic tissues (no green or blue collagen tissue was seen in the injection site). (C-F) Transplanted TA muscle was stained with anti-GFP (Alexa 488) (D), connexin 43 (Alexa 594) (E) and DAPI (F, merged with DAPI). (C) Montage of the pCPCs transplanted tissue along the TA muscle identified as GFP+ and connexin 43 positive. (D-F) Higher magnification of transplanted tissue showed connexin 43 expression at cell-cell membrane boundaries. (G) Masson's trichrome staining of transplanted TA muscle of over $100 \mu \mathrm{m}$ distant to (A) also showed transplanted pCPCs in the injection track. (I) An adjacent slide of area depicted by arrowhead was positive for cardiac Troponin-T Alexa 647. I. In this area transplanted pCPCs identified as GFP+ were also connexin 43 positive. Scale bars $=100 \mu \mathrm{m}$.
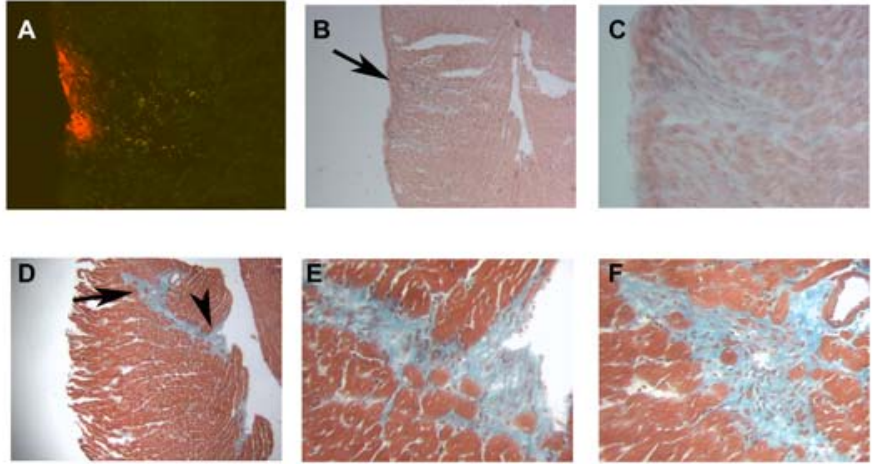

GFP

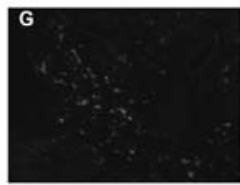

PKH26
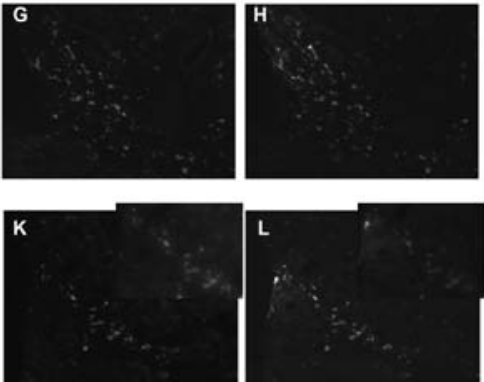
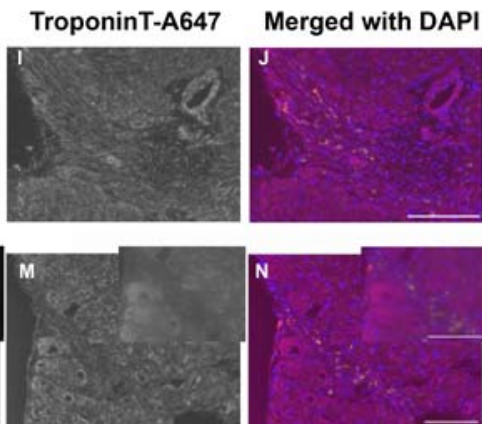

Figure 7: pCPCs transplanted in the heart did not express cardiac troponin and were found in fibrotic tissue. (A) Transplanted pCPCs identified as GFP+/ $\mathrm{PKH} 26+$ cells (green and red fluorescent, respectively) were located in the left ventricular wall. The bright red florescence emitted from PKH26 greatly facilitated the screening of the heart tissue to locate the transplanted cells. (B) H\&E staining showed fibrotic-like tissue in the transplanted area. (C) Higher magnification of area pointed by an arrow in (B). (D) Masson's trichrome staining showed collagen deposition (in blue) in the transplanted area. (E) Higher magnification of area pointed by an arrowhead in (D). (F) Higher magnification of area pointed by arrow in (D). (G-J) Transplanted pCPCs identified as GFP+/PKH26+ did not exhibit cardiomyocyte morphology and did not stain positive for cardiac Troponin T. (K-N) Another transplanted heart clearly showed GFP+/PKH26+ cells in the injection track were negative for cardiac Troponin T (M-N insets). Scale bars $=100 \mu \mathrm{m}$ 
$\mathrm{Nkx} 2.5$ and c-kit in culture indicates either commitment of endogenous cardiac stem cells into cardiac progenitor cells or an in vitro artifact resulting in expression of these cardiac progenitor cell markers in a cell population that does not have endogenous cardiogenic function. The pCPCs isolated with our protocol did not differentiate into endothelial cells or hepatocytes using the same protocols previously reported for differentiation of multipotent adult progenitor cells (MAPC) from the bone marrow $[19,20]$, suggesting that the pCPCs isolated with our method from the adult atrium are committed to the cardiac cell fate. This unipotentiality or restriction to the cardiac lineage was also observed when pCPCs were transplanted in the skeletal muscle resulting only in cardiac-like cells. Additionally, although the pCPCs expressed some of the pluripotent transcription factors, such as Nanog and KLF4, they did not express the Oct4 pluripotent marker which may explain their limited differentiation capacity.

Sca-1 positive pCPCs did not express c-kit at the beginning of culture indicating absence of c-kit positive cells immediately in culture. This result is the same as previously reported by $\mathrm{Oh}$ et al. [8]. Because c-kit ${ }^{+}$cardiac progenitor cells decline rapidly after birth [30], we hypothesize that our culture conditions either select for a very small c-kit+ population that outgrows in culture, or that these culture conditions induce dedifferentiation. The latter is unlikely, since the pCPCs generated under these culture conditions only differentiated into cardiac-like cells and not into cells of any other lineage.

Most of the protocols reported to induce beating cardiomyocytes have used co-culture methods with mature cardiomyocytes [10] or induction with 5-aza-deoxycytidine (5-azadC), an inhibitor of DNA methylation previously reported to promote the differentiation of pluripotent P19 embryonal carcinoma cells [8,9]. We induced cardiomyocytes differentiation with oxytocin treatment in DMEM plus $10 \%$ FBS for 21 days, which showed expression of cardiac proteins by immunohistochemistry staining and cardiac-specific genes by RT-PCR. However, the phenotype of oxytocin-induced pCPCs was immature and no beating cells were observed in our cultures. In contrast to the studies reported by Matsuura et al. [18], which resulted in beating cardiomyocytes when induced with oxytocin, we cultured the cells for a shorter period of time and we did not replate the cells at confluence.

Nonetheless, pCPCs transplanted into the skeletal muscle formed muscle bundles that expressed mature cardiac proteins, suggesting these cells turned into mature cardiomyocytes. Furthermore, pCPCs that generated cardiac muscle bundles in the skeletal muscle stained positive for connexin 43 at cell-cell boundaries across the transplanted tissue, indicating that pCPCs differentiated into cardiac muscle bundles with gap junctions. In contrast, pCPCs transplanted into the heart in vivo did not differentiate into cardiomyocytes, but instead, resulted in fibrosis at the site of injection. This finding lands further evidence to the idea that the adult heart may not be a very permissive environment for cardiomyogenesis, and in turn, the heart's response to injury is scar formation [16,31]. In light of the fact that the heart-derived pCPCs did differentiate into cardiomyocyte-like cells in skeletal muscle, further studies may be warranted investigating the endogenous factors in the skeletal muscle that permit cardiogenesis and may be missing in the heart environment. Identification of such cardiogenesis-permitting growth factors could open up a new venue for strategies to coax the injured heart into regeneration with exogenous growth factor therapy. Although, transplantation of the GFP+ cells into the wild type host cannot rule out cell fusion, the lack of expression of skeletal muscle specific myosin in the transplanted pCPCs excludes fusion in the skeletal muscle transplants.

One of the limitations of the present study is the absence of determination of the identity of the cell type capable of becoming pCPC in culture. Future studies should aim at prospectively isolating pCPCs using stem cell markers such as c-kit and Sca-1. Unfortunately, humans do not have the Sca-1 gene [32,33]. Although we have not yet determined if similar cardiac progenitor cells can be derived from the human heart, our method, which does not depend on any cell surface markers, may contribute to a protocol for human CPCs culture for future cell-based therapies.

\section{Acknowledgements}

We will like to thank Dr. Michael Preusch for performing the intra-cardiac transplants. Funding for this research has been provided by the Departments of Pathology and Lab Medicine at the University of Washington, and Perkins Coie Award.

\section{References}

1. Heron M, Hoyert DL, Murphy SL, Xu J, Kochanek KD, et al. (2009) Deaths: Final data for 2006. Natl Vital Stat Rep 57: 1-134.

2. Poston RS, Griffith BP (2004) Heart transplantation. J Intensive Care Med 19 3-12

3. Radovancevic B, Frazier OH (1999) Heart transplantation: approaching a new century. Tex Heart Inst J 26: 60-70.

4. Laflamme MA, Zbinden S, Epstein SE, Murry CE (2007) Cell-based therapy for myocardial ischemia and infarction: pathophysiological mechanisms. Annu Rev Pathol 2: 307-339.

5. Hsieh PC, Segers VF, Davis ME, MacGillivray C, Gannon J, et al. (2007) Evidence from a genetic fate-mapping study that stem cells refresh adult mammalian cardiomyocytes after injury. Nat Med 13: 970-974.

6. Bergmann O, Bhardwaj RD, Bernard S, Zdunek S, Barnabe-Heider F, et al (2009) Evidence for cardiomyocyte renewal in humans. Science 324: 98-102.

7. Beltrami AP, Barlucchi L, Torella D, Baker M, Limana F, et al. (2003) Adult cardiac stem cells are multipotent and support myocardial regeneration. Cell 114: 763-776.

8. Oh H, Bradfute SB, Gallardo TD, Nakamura T, Gaussin V, et al. (2003) Cardiac progenitor cells from adult myocardium: homing, differentiation, and fusion after infarction. Proc Natl Acad Sci U S A 100: 12313-12318.

9. Wang X, Hu Q, Nakamura Y, Lee J, Zhang G, et al. (2006) The role of the sca-1+/CD31- cardiac progenitor cell population in postinfarction left ventricular remodeling. Stem Cells 24: 1779-1788.

10. Pfister O, Mouquet F, Jain M, Summer R, Helmes M, et al. (2005) CD31- but Not CD31+ cardiac side population cells exhibit functional cardiomyogenic differentiation. Circ Res 97: 52-61.

11. Pfister O, Oikonomopoulos A, Sereti KI, Liao R (2010) Isolation of resident cardiac progenitor cells by Hoechst 33342 staining. Methods Mol Biol 660: 5363.

12. Moretti A, Caron L, Nakano A, Lam JT, Bernshausen A, et al. (2006) Multipotent embryonic isl1+ progenitor cells lead to cardiac, smooth muscle, and endothelial cell diversification. Cell 127: 1151-1165.

13. Lam ML, Hashem SI, Claycomb WC Embryonic stem cell-derived cardiomyocytes harbor a subpopulation of niche-forming Sca-1+ progenitor cells. Mol Cell Biochem 349: 69-76.

14. Fazel S, Cimini M, Chen L, Li S, Angoulvant D, et al. (2006) Cardioprotective c-kit+ cells are from the bone marrow and regulate the myocardial balance of angiogenic cytokines. J Clin Invest 116: 1865-1877.

15. Jackson KA, Majka SM, Wang H, Pocius J, Hartley CJ, et al. (2001) Regeneration of ischemic cardiac muscle and vascular endothelium by adult stem cells. J Clin Invest 107: 1395-1402. 
Citation: Reesukumal K, Pratumvinit B, Rudakova M, Janebodin K, Reyes M (2011) Purification, Expansion and Characterization of Putative Murine Cardiac Progenitor Cells . J Stem Cell Res Ther S1:002. doi:10.4172/2157-7633.S1-002

16. Li Z, Lee A, Huang M, Chun H, Chung J, et al. (2009) Imaging survival and function of transplanted cardiac resident stem cells. J Am Coll Cardiol 53: 12291240.

17. Jiang Y, Jahagirdar BN, Reinhardt RL, Schwartz RE, Keene CD, et al. (2002) Pluripotency of mesenchymal stem cells derived from adult marrow. Nature 418: 41-49.

18. Matsuura K, Nagai T, Nishigaki N, Oyama T, Nishi J, et al. (2004) Adult cardiac Sca-1-positive cells differentiate into beating cardiomyocytes. J Biol Chem 279: 11384-11391.

19. Reyes M, Dudek A, Jahagirdar B, Koodie L, Marker PH, et al. (2002) Origin of endothelial progenitors in human postnatal bone marrow. J Clin Invest 109: 337-346.

20. Schwartz RE, Reyes M, Koodie L, Jiang Y, Blackstad M, et al. (2002) Multipotent adult progenitor cells from bone marrow differentiate into functional hepatocyte-like cells. J Clin Invest 109: 1291-1302.

21. Eppenberger-Eberhardt M, Flamme I, Kurer V, Eppenberger HM (1990) Reexpression of alpha-smooth muscle actin isoform in cultured adult rat cardiomyocytes. Dev Biol 139: 269-278.

22. Fleischmann M, Bloch W, Kolossov E, Andressen C, Muller M, et al. (1998) Cardiac specific expression of the green fluorescent protein during early murine embryonic development. FEBS Lett 440: 370-376.

23. Clement S, Stouffs M, Bettiol E, Kampf S, Krause KH, et al. (2007) Expression and function of alpha-smooth muscle actin during embryonic-stem-cell-derived cardiomyocyte differentiation. J Cell Sci 120: 229-238.

24. Ruzicka DL, Schwartz RJ (1988) Sequential activation of alpha-actin genes during avian cardiogenesis: vascular smooth muscle alpha-actin gene transcripts mark the onset of cardiomyocyte differentiation. J Cell Biol 107 2575-2586.
25. Allwork SP, Bentall HH (1986) Usefulness of the phenomenon of histofluorescence in the identification of early myocardial necrosis. Cardiovasc Res 20: 451-457.

26. Ford JW, Welling TH 3rd, Stanley JC, Messina LM (1996) PKH26 and 125I-PKH95: characterization and efficacy as labels for in vitro and in vivo endothelial cell localization and tracking. J Surg Res 62: 23-28.

27. D'Ippolito G, Diabira S, Howard GA, Menei P, Roos BA, et al. (2004) Marrowisolated adult multilineage inducible (MIAMI) cells, a unique population of postnatal young and old human cells with extensive expansion and differentiation potential. J Cell Sci 117: 2971-2981.

28. D'Ippolito G, Howard GA, Roos BA, Schiller PC (2006) Isolation and characterization of marrow-isolated adult multilineage inducible (MIAMI) cells. Exp Hematol 34: 1608-1610.

29. Beltrami AP, Cesselli D, Bergamin N, Marcon P, Rigo S, et al. (2007) Multipotent cells can be generated in vitro from several adult human organs (heart, liver and bone marrow). Blood 110: 3438-3446.

30. Tallini YN, Greene KS, Craven M, Spealman A, Breitbach M, et al. (2009) C-kit expression identifies cardiovascular precursors in the neonatal heart. Proc Natl Acad Sci U S A 106: 1808-1813.

31. Frangogiannis NG (2006) The mechanistic basis of infarct healing. Antioxid Redox Signal 8: 1907-1939.

32. Kamiura S, Nolan CM, Meruelo D (1992) Long-range physical map of the Ly- 6 complex: mapping the Ly- 6 multigene family by field-inversion and twodimensional gel electrophoresis. Genomics 12: 89-105.

33. Holmes C, Stanford WL (2007) Concise review: stem cell antigen-1: expression, function, and enigma. Stem Cells 25: 1339-1347.
This article was originally published in a special issue, Cardiovascular Cell Therapy handled by Editor(s). Dr. Martin Rodriguez-Porcel, Medicine Mayo Medical School, USA; Dr. Rosalinda Madonna, USA 